Results: From 2,644 citations identified through electronic search, ten studies met the inclusion criteria. According to the studies mode of delivery influences oral microbial density, oral microbial profile and the timing of oral colonization by cariogenic microbiota. However, there are no consistent results concerning either the prevalence of children harboring cariogenic microbiota or the prevalence of early childhood caries by mode of delivery.

Conclusion: Mode of delivery influences early oral colonization. However, it seems that other determinants rather than mode of delivery could be major contributors to the development of early childhood caries.

\title{
OC29 - Caregiver burden in childhood asthma
}

\author{
Ayfer Ekim (Turkey) ${ }^{1}$ \\ ${ }^{1}$ Istanbul Bilgi University School of Health Sciences Department of Nursing
}

Theme: Parenting/parenthood.

Keywords: Burden, caregiver, childhood asthma, parent.

Introduction: As delivering care is an inherent part of becoming parents, providing highlevel care to the child with health problems might be a burden for the caregiver.

\begin{abstract}
Aim: This systematic review investigated the effects of caregiver burden of parents who are primarily responsible for the caring of children with asthma.
\end{abstract}

Methods: PubMed, CINAHL, Web of Science and Scopus databases were searched for the studies published between 2000-2015. Key search terms used were 'caregiver', 'burden, 'childhood asthma' and 'parent'.

Results: A total of 23 studies were examined for this review.

Discussion: Providing care to the child with asthma affects parents as caregivers negatively. Caregivers of the children with asthma often experience anxiety, fear, disappointment, grief, physical distress and low quality of life as the outcomes of caregiving burden.

Conclusions: It is absolutely crucial to determine caregiver burden risks and protective factors since wellness level of caregivers affect the asthma management practices and decisions.

\section{OC30 - Fracture reduction with nitrous oxide at the children's emergency department shortens the length of stay and reduces the use of full anaesthesia in the operating department}

Sigrid Lang (Sweden)ㄹ; Anna-Pia Wentzel (Sweden)루

Malin Ekstrom (Sweden) ${ }^{1}$

${ }^{1}$ Queen Silvias Hospital, Gothenburg

Theme: Accreditation and quality improvement. 
Keywords: Nitrous oxide, length of stay, fracture reduction.

Aim: Dislocated fractures are common in the children's emergency department (ER). All forms of fracture reduction are very painful requiring nitrous oxide. The purpose is to shorten the length of stay in the hospital as well as sustain a high quality of care.

Methods: All nurses received theoretical and practical training in the use of nitrous oxide. Evaluations with the families were made by telephone.

Result: A total of 40 enclosed fracture reductions were made at the ER, leading to a reduction of 33 patients in the operating department and the length of stay was shortened this compared to the same time in 2014.

No adverse event was reported and no patient felt any increase in pain during the treatment. All patients would repeat the procedure if necessary.

Conclusion: The treatment has reduced the length of stay in the hospital without affecting the other patients in the ER or the quality of care.

\title{
OC31 - Tobacco consumption by adolescents: a study in the north of Portgual
}

\author{
Margarida Reis Santos (Portugal) ${ }^{1}$; Sandra Cruz (Portugal) ${ }^{1}$; \\ Susana Fernandes (Portugal)2
}

${ }^{1}$ ESEP; ${ }^{2}$ ULSNE - UCC Mogadouro

Theme: School health.

Keywords: Adolescents, risk behaviours, tobacco.

Aims: To characterize the adolescents' habits of smoking in a Portuguese School; to identify if the adolescents have information on the harmful effects of tobacco and their health education needs.

Methods: Quantitative, exploratory and descriptive study. Data collection in 2011 through a self-answered questionnaire.

Results: The sample comprised 441 students; mean age of 14.5 years old; $53.1 \%$ were male; $18,6 \%$ were smokers; boys smoked more than girls; they started to smoke between 7-18; the tobacco consumption is significantly related with: age $(p<0,001)$; gender $(p=0,002)$; interest of the family about the adolescents' problems $(p=0,028)$ among others. The adolescents have information about the harmful effects of tobacco and the ones interested in receiving more information wanted it from the health professionals.

Conclusions: Prevention and delaying the age of initiation of consumption should be taken into account in a target intervention plan, which should be led by the school health nurse. 